\title{
Aprendizaje basado en problemas multiprofesional: Estudio cualitativo desde la perspectiva de los tutores
}

\author{
Nancy Navarro $H^{1 a}$, Mónica Illesca $P^{2 b}$, \\ Mirtha Cabezas $\mathbf{G}^{3 \mathrm{C}}$. \\ Problem based learning from the
perspective of tutors
}

Background: Problem based learning is a student centered learning technique that develops deductive, constructive and reasoning capacities among the students. Teachers must adapt to this paradigm of constructing rather than transmitting knowledge. Aim: To interpret the importance of tutors in problem based learning during a module of Health research and management given to medical, nursing, physical therapy, midwifery, technology and nutrition students. Material and methods: Eight teachers that participated in a module using problem based learning accepted to participate in an in depth interview. The qualitative analysis of the textual information recorded, was performed using the ATLAS software. Results: We identified 662 meaning units, grouped in 29 descriptive categories, with eight emerging meta categories. The sequential and cross-generated qualitative analysis generated four domains: competence among students, competence of teachers, student-centered learning and evaluation process. Conclusions: Multiprofessional problem based learning contributes to the development of generic competences among future health professionals, such as multidisciplinary work, critical capacity and social skills. Teachers must shelter the students in the context of their problems and social situation (Rev Méd Chile 2009; 137: 246-54).

(Key words: Health occupations; Manager care programs; Problem-based learning)

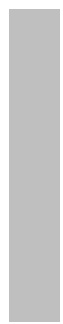

\footnotetext{
Recibido el 23 de junio, 2008. Aceptado el 25 de noviembre, 2008.

Trabajo financiado por Proyecto DIUFRO 120538 Vicerrectoría de Investigación y Postgrado Dirección de Investigación, Universidad de La Frontera, Temuco, Chile.

${ }^{1}$ Oficina de Educación en Ciencias de la Salud (OFECS). Departamentos de Obstetricia y Ginecología, ${ }^{2}$ Medicina Interna. ${ }^{3}$ Ciencias Preclínicas, Facultad de Medicina, Universidad de La Frontera, Temuco, Chile.

aMatrona, Magíster en Pedagogía y Gestión Universitaria

benfermera, Magíster en Pedagogía y Gestión Universitaria

${ }^{\mathrm{C}} \mathrm{Químico}$ Farmacéutico, Diplomada en Pedagogía y Gestión Universitaria
}

Correspondencia a: Nancy Navarro Hernández. Oficina de Educación en Ciencias de la Salud (OFECS). Departamento Obstetricia y Ginecología, Facultad de Medicina, Universidad de La Frontera. Casilla 54 D Temuco, Chile. Fono: 45-325705. Fax: 45-325710. E mail: nnavarro@ufro.cl 
E n la formación de los profesionales de la salud se conjugan tanto los lineamientos ministeriales de educación y salud, como las demandas de un mundo cambiante y globalizado. Ello implica, para todos los involucrados en este proceso, comprometerse con la nueva concepción de las prácticas educativas, es decir, trabajar en base a problemas prioritarios de salud y contribuir a desarrollar competencias genéricas.

Para el logro de estos objetivos se postula que el proceso educativo debe estar centrado en el estudiante, con principios de educación del adulto, para un aprendizaje significativo y desarrollo de competencias genéricas: instrumentales, interpersonales y sistémicas ${ }^{1,2}$. Todo ello, además, es concordante con las políticas de salud, que establecen fortalecer el trabajo multiprofesional, intersectorial en los tres niveles de atención.

En este contexto, los modelos de formación adquieren importancia, abocados a la mejora de la práctica educativa, principalmente, lo concerniente al desempeño docente y factores que interactúan en el proceso educativo. El modelo crítico-reflexivo, que forma parte de un movimiento de renovación curricular y de enseñanza más amplia, orientado a la indagación centrada en la investigación crítica y atendiendo a las causas y consecuencias de las acciones en el aula, donde los docentes tienen la capacidad intelectual de valorar su propia práctica ${ }^{3}$. Enfatiza el aprendizaje, reconoce en el estudiante la capacidad para plantear problemas, reflexionando a partir de su propia acción en colaboración con otros, discutiendo, resolviendo temáticas, construyendo conocimientos a través de sus experiencias ${ }^{4}$. En resumen, el adoptar un modelo de formación implica un cambio de comportamiento continuo, tanto para el educando como para el educador.

En este contexto, los planes de estudio de la innovación curricular de la Facultad de Medicina, Universidad de La Frontera desde 2003, privilegia la didáctica activa centrada en el estudiante, fortaleciendo las competencias genéricas conjuntamente a las específicas de la profesión.

Este cambio educativo ha considerado una línea transversal, multiprofesional durante los dos primeros años, donde se trabaja, fundamentalmente en aprendizaje basado en problemas (ABP) en grupos pequeños. Las situaciones de estudio son temas prioritarios de salud con una visión holística e integración de las diferentes disciplinas, con un fuerte componente ético-humanista.

El ABP no es una estrategia de aprendizaje rígida, varios son los autores que intentan definirlo ${ }^{5-9}$, para ellos es una actividad constructiva, "aprender haciendo", una forma de enseñanza deductiva, una conjunción de la teoría y la práctica que permite utilizar estrategias de razonamiento para combinar y sintetizar datos, en la cual el punto de partida es un problema lo más cercano a la realidad donde el educando, mediante la participación activa en el grupo, identifica necesidades e integra conocimientos utilizando múltiples recursos.

Esta didáctica se basa en el constructivismo 10 $\mathrm{y}$ favorece los cuatro pilares educativos ${ }^{11}$ : del saber, del saber hacer, del saber convivir y del saber ser. Ello conlleva un cambio de actitud tanto del docente como del estudiante. Para el primero, implica una manera distinta de entender el proceso de enseñanza-aprendizaje, no es la mera transmisión de conocimiento ya elaborado, sino más bien la construcción de éste. Para el segundo, significa ser protagonista y gestor del proceso educativo, aplicar el método científico y la toma de decisiones a través del análisis crítico, desarrollar potencialidades personales, favorecer la autonomía a través de la reflexión sobre cómo construyen sus propios aprendizajes, estimular el desarrollo de la creatividad, favorecer entre otras el autoaprendizaje, autoformación, objetividad para la autoevaluación, habilidades para comunicarse, relaciones interpersonales, aprendizaje colaborativo y trabajo grupal.

Para los académicos involucrados en la innovación curricular desarrollada en la Facultad de Medicina ha significado asumir un nuevo rol en el quehacer de la práctica educativa en diversas dimensiones y niveles en el proceso de cambio ${ }^{12}$. De acuerdo a ello, Fullan enmarca el cambio desde lo más simple y superficial (a corto plazo) hasta lo más complejo y profundo (a largo plazo), el cual afecta a las creencias, valores e ideología que modelan las concepciones y prácticas pedagógicas $^{13}$.

Dentro de las diversas dimensiones, se ha considerado en este estudio establecer una relación con las competencias descritas por Scriven y Jiménez"14,15 en relación a: "qué debe saber el docente" (construcción teórica y metodológica de 
la práctica educativa centrada en el estudiante), "qué debe saber hacer" (competencias didácticas, trabajo en grupo pequeño), "cómo debe ser y comportarse" (relaciones interpersonales, habilidades sociales) y "saber aprender" (participación en proyectos de mejora de la calidad docente, a los procesos de autorregulación de la propia práctica y al trabajo colegiado con otros académicos).

Dada la naturaleza del estudio, comprender en profundidad el significado que tiene para los docentes el aprendizaje basado en problemas multiprofesional, se formulan los siguientes objetivos orientadores:

- Indagar las competencias que desarrollan los estudiantes con el aprendizaje basado en problemas multiprofesional.

- Explorar las competencias del profesorado en la metodología del aprendizaje basado en problemas multiprofesional.

- Analizar características del aprendizaje.

\section{Material y MÉTODO}

Se plantea una investigación educativa desde el paradigma cualitativo con enfoque fenomenológico, hermenéutico ${ }^{16-19}$ a través de un estudio intrínseco de caso $^{20}$, es decir, comprensión de la conducta humana en un marco de referencia particular, describiendo e interpretando el fenómeno a indagar.

En lo particular, el estudio intrínseco de casos permite profundizar la comprensión del fenómeno en estudio: comprender en profundidad el significado que tiene para los docentes el aprendizaje basado en problemas multiprofesional. En lo concreto, el caso intrínseco es la experiencia educativa del Módulo de Gestión e Investigación en Salud I, desarrollada en grupo pequeño multiprofesional, con metodología de aprendizaje basado en problemas impartido a estudiantes de primer año de las carreras de: Enfermería, Kinesiología, Medicina, Nutrición, Obstetricia y Tecnología Médica de la Facultad de Medicina, Universidad de La Frontera.

La población en estudio son los tutores que participaron en esta actividad educativa (2006), quienes a partir de sus historias personales individuales y colectivas proporcionaron información válida con relación al propósito de la investigación.

La muestra, de tipo no probabilística, fue intencionada de casos por criterios, conformada por ocho académicos de diferentes profesiones de la salud
(Enfermería, Obstetricia y Puericultura, Kinesiología, Medicina, Nutrición, Tecnología Médica, Químico Farmacéutico), cuyo criterio de inclusión fue haber participado en la experiencia educativa (GIS I), quienes aceptaron su participación mediante la firma de un consentimiento informado, garantizando el anonimato y la confidencialidad de los datos.

La recolección de datos se obtuvo mediante la técnica de entrevista en profundidad, realizadas durante el segundo semestre académico de 2006, guiada por un facilitador, quien contó con una pauta para indagar las temáticas del estudio enmarcadas en los objetivos de la investigación, en una o dos sesiones con un máximo de tiempo de hora y media, hasta llegar al punto de saturación de los datos. Esta fase y el análisis de datos ocurrieron simultáneamente a lo largo de todo el proceso.

Como técnica de registro de información se emplearon notas de campo y grabaciones magnetofónicas, realizándose transcripciones literales con el objetivo de recoger las palabras exactas de los informantes. Las transcripciones fueron devueltas a los participantes para su revisión y aprobación.

El análisis de datos fue el de comparaciones constantes de Glaser y Strauss ${ }^{21}$, no agrupándose en categorías predeterminadas, sino más bien emergiendo de un proceso de razonamiento inductivo, es un método generativo, constructivo e inductivo en el que se combina la codificación inductiva de categorías con la comparación constante de ellas. Se adoptó el esquema propuesto por Miles y Huberman ${ }^{22}$, a través de la reducción progresiva (separación de unidades, agrupamiento, identificación y clasificación de elementos), disposición, transformación y obtención de conclusiones verificables. Como herramienta computacional se utilizó el programa ATLAS - ti.

El nivel progresivo de reducción y estructuración de los datos fue a través de los siguientes niveles:

- Nivel 1: segmentación e identificación de unidades de significado (codificación) y agrupación en categorías descriptivas de los fragmentos de texto con sentido semántico (perspectiva hermenéutica y émica relacionada directamente con el carácter reflexivo desde el punto de vista del informante).

- Nivel 2: construcción de un sistema de núcleos temáticos emergentes o metacategorías (perspectiva etic, dimensión interpretativa del investigador, a partir de los conceptos del primer nivel). 
- Nivel 3: identificación de dominios cualitativos (análisis secuencial y transversal de las metacategorías).

La rigurosidad científica se garantizó utilizando los criterios de rigor $^{23}$ : credibilidad (triangulación por investigador, comprobación con los participante), dependencia (réplica paso a paso que evidencia el trabajo realizado permitiendo juzgar el grado de correspondencia con otros contextos), confirmabilidad (ejercicio de reflexión y consenso con otros investigadores) y transferibilidad (recogida de abundante información descriptiva hasta la saturación de los datos y desarrollo de descripciones minuciosas).

\section{Resultados}

En el nivel progresivo de reducción "Nivel 1" se identificaron 662 unidades de significado relevantes (conceptos o palabras que hacen referencia a un mismo tópico), agrupadas en 29 categorías descriptivas emergentes, las que se presentan en la Tabla 1.

Posterior al primer nivel se procede a la reducción de datos en el Nivel 2, donde se agruparon las 29 categorías descriptivas, emergiendo 8 metacategorías las que se presentan en la Tabla 2.

Tabla 1. Unidades de significado por categorías descriptivas de acuerdo a las entrevistas

\begin{tabular}{|c|c|c|c|c|c|c|c|c|c|c|}
\hline \multirow[b]{2}{*}{ No } & \multirow[b]{2}{*}{ Categorías descriptivas } & \multicolumn{9}{|c|}{ Entrevistas con unidades de significado } \\
\hline & & 1 & 2 & 3 & 4 & 5 & 6 & 7 & 8 & Total \\
\hline 1. & Aprender en forma activa & - & - & 1 & - & - & 4 & 7 & 4 & 16 \\
\hline 2. & Aprender colaborativamente & 2 & - & 1 & 8 & - & 3 & 2 & 1 & 17 \\
\hline 3. & Integrar conocimientos & 1 & - & 3 & - & 2 & - & 1 & 1 & 8 \\
\hline 4. & Aprender en forma significativa & 3 & 1 & 5 & - & - & 1 & 1 & 1 & 12 \\
\hline 5. & Capacidad priorización del estudiante & - & 2 & 2 & 2 & - & 2 & 5 & - & 13 \\
\hline 6. & Relaciones interpersonales & - & - & 1 & 7 & 2 & 1 & 1 & - & 12 \\
\hline 7. & Trabajo en equipo multiprofesional & 14 & 13 & 10 & 9 & 7 & 3 & 3 & 1 & 60 \\
\hline 8. & Estudiante participativo & 8 & 1 & 3 & 1 & 4 & 2 & - & 1 & 20 \\
\hline 9. & Autonomía estudiantil & 2 & 5 & 7 & 6 & 6 & 1 & 7 & 4 & 38 \\
\hline 10. & Capacidad de análisis crítico & 2 & 10 & 4 & 8 & 4 & 5 & 2 & 2 & 37 \\
\hline 11. & Liderazgo & - & 4 & 2 & - & - & - & - & 1 & 7 \\
\hline 12. & Ambiente aprendizaje favorable & 6 & 3 & 7 & 3 & 3 & - & - & - & 22 \\
\hline 13. & Desarrollo personal & - & - & 8 & - & 5 & - & 1 & - & 14 \\
\hline 14. & Desarrollo valórico & - & 6 & 10 & 6 & 1 & - & 3 & 4 & 30 \\
\hline 15. & Compromiso docente & 5 & 3 & 4 & 3 & 2 & - & 4 & - & 21 \\
\hline 16. & Docente facilitador del aprendizaje & 5 & 3 & 5 & 6 & 3 & - & 8 & 3 & 33 \\
\hline 17. & Docente facilitador de la evaluación & 3 & 3 & 4 & 1 & 2 & - & 1 & - & 14 \\
\hline 18. & Facilitador ambiente aprendizaje & 5 & 3 & 3 & 6 & 3 & 3 & 3 & 1 & 27 \\
\hline 19. & Conocimiento de la disciplina & 1 & - & - & - & - & - & 3 & - & 4 \\
\hline 20. & Dominio metodológico & 6 & 4 & 8 & 4 & 6 & 10 & 3 & 7 & 48 \\
\hline 21. & Autoevaluación estudiantil & 2 & 3 & 1 & 7 & 1 & 2 & 4 & 5 & 25 \\
\hline 22. & Autoevaluación docente & 9 & 5 & - & 2 & 4 & - & 1 & 1 & 22 \\
\hline 23. & Evaluación de pares & 2 & 2 & 4 & 6 & 2 & 2 & 3 & 2 & 23 \\
\hline 24. & Evaluación a docente & 3 & 5 & 4 & 3 & 1 & 2 & 2 & 5 & 25 \\
\hline 25. & Evaluación compleja & 4 & 10 & 9 & 4 & 3 & 7 & 4 & 1 & 42 \\
\hline 26. & Evaluación significativa & 1 & 5 & 6 & 7 & 5 & - & - & 3 & 27 \\
\hline 27. & Habilidades comunicacionales estudiante & 2 & - & 5 & 1 & 1 & 4 & 2 & 2 & 17 \\
\hline 28. & Habilidades comunicacionales docente & 1 & - & 1 & 1 & 3 & - & 1 & 1 & 8 \\
\hline \multirow[t]{2}{*}{29.} & Trabajo en equipo docente multiprofesional & 12 & 2 & 2 & - & 3 & - & 1 & - & 20 \\
\hline & Total & 99 & 93 & 120 & 101 & 73 & 52 & 73 & 51 & 662 \\
\hline
\end{tabular}


Tabla 2. M etacategorías, con sus correspondientes categorías y unidades de significado

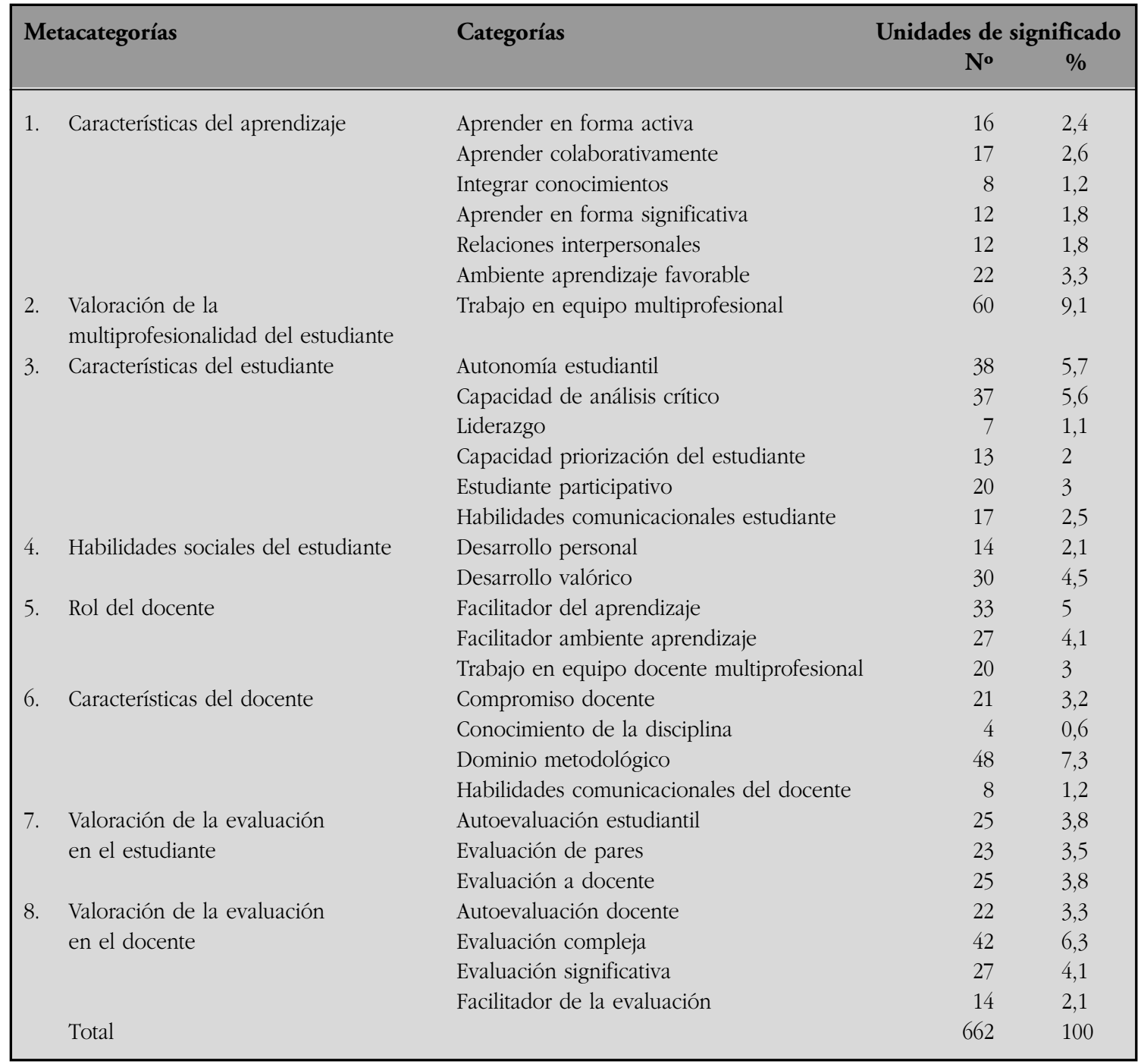

Finalmente en la reducción de datos del Nivel 3, emergen 4 dominios cualitativos: "Competencias en el estudiantado", "Competencias del profesorado", "Aprendizaje centrado en el estudiante" y "Proceso evaluativo".

Para objetivizar la reducción de datos se presenta en la Tabla 3 el resumen comparativo.

Las Tablas 4 a 7 describen cada dominio con las unidades de significado más representativas.
DISCUSIÓN

Los resultados de este estudio evidencian que el ABP multiprofesional contribuye al desarrollo de las competencias genéricas de los futuros profesionales de la salud, planteadas tanto por el Tuning ${ }^{1}$ como la Universidad de La Frontera ${ }^{2}$. Por otra parte, el estudiantado con esta estrategia metodológica desarrolla la capacidad crítica al 
Tabla 3. Comparación porcentual entre dominios, metacategorías, categorías y unidades de significado

\begin{tabular}{|c|c|c|c|c|c|c|}
\hline \multicolumn{4}{|c|}{ Nivel 1} & \multirow{2}{*}{\multicolumn{2}{|c|}{$\begin{array}{c}\text { Nivel } 2 \\
\text { Metacategorías }\end{array}$}} & \multirow{3}{*}{$\begin{array}{c}\text { Nivel } 3 \\
\text { Dominios }\end{array}$} \\
\hline \multicolumn{2}{|c|}{ Unidades de significado } & \multicolumn{2}{|c|}{ Categorías } & & & \\
\hline No & $\%$ & No & $\%$ & No & $\%$ & \\
\hline 236 & 35,6 & 9 & 31,1 & 3 & 37,5 & Dominio 1 \\
\hline 161 & 24,3 & 7 & 24,1 & 2 & 25 & Dominio 2 \\
\hline 87 & 13,2 & 6 & 20,7 & 1 & 12,5 & Dominio 3 \\
\hline 178 & 26,9 & 7 & 24,1 & 2 & 25 & Dominio 4 \\
\hline 662 & 100 & 29 & 100 & 8 & 100 & Total \\
\hline
\end{tabular}

Tabla 4. Descripción del dominio "Competencias en el estudiantado" con unidades de significado representativas

\begin{tabular}{|c|c|}
\hline Dominio 1: "Competencias en el estudiantado" & Unidades de significado representativas \\
\hline $\begin{array}{l}\text { Opiniones en relación al desarrollo de } \\
\quad \text { competencias genéricas: } \\
\text { - } \text { Autonomía } \\
\text { - } \text { Análisis crítico } \\
\text { - } \text { Proactividad } \\
\text { - Habilidades comunicacionales } \\
\text { - Capacidad de priorización } \\
\text { - } \text { Aprendizaje multiprofesional } \\
\text { - Desarrollo personal y valórico }\end{array}$ & $\begin{array}{l}\text { "aprenden a ser independientes, autónomos, capa- } \\
\text { ces de buscar y resolver problemas e inquietudes", } \\
\text { "ellos se autorregulan" } \\
\text { "analíticos, se replantean, buscan apoyo" } \\
\text { "crean su aprendizaje de acuerdo a sus habilidades } \\
\text { y solicitan ayuda al grupo" } \\
\text { "desarrollan habilidad de diálogo, respeto", "mejo- } \\
\text { ran timidez y dificultad de lenguaje..." } \\
\text { "profundizan lo que les interesa" } \\
\text { "generan respeto por otros profesionales, conocen } \\
\text { otras profesiones, roles", "la multiprofesional mues- } \\
\text { tra una visión que no es lograda por si sola" } \\
\text { "manejan sus límites, sintiéndose grandes, logrando } \\
\text { madurez" } \\
\text { "aprenden a ser tolerantes, respetuosos, normas, } \\
\text { hábitos", "desarrollan actitudes de responsabilidad, } \\
\text { escuchar y valorar al otro" }\end{array}$ \\
\hline
\end{tabular}

realizar un aprendizaje activo, significativo, integrado y colaborativo a través de la construcción de conocimiento en forma conjunta, coincidente con lo planteado en la literatura ${ }^{5-9}$.

Los docentes destacan el trabajo multiprofesional, se establecen relaciones de horizontalidad y colaboración comprendiendo el rol de las otras profesiones de la salud. Ello implica una mayor cohesión y solidaridad entre los estudiantes, generando un clima grato de estudio, modelo que podría ser replicado en el futuro laboral. Otro aspecto identificado es el desarrollo de habilidades sociales, lo que permite la formación de una persona integral, rol que le compete a las instituciones educativas como centro formador.

Los docentes en este estudio mencionan que el ABP multiprofesional favorece un clima de confianza, facilita el proceso de aprendizaje, entrega valores y permite realizar crítica constructiva. Sin embargo, expresan que para lograr el aprendizaje efectivo, este proceso de facilitación no sólo se refiere al hecho de adquirir contenidos, 


\section{Tabla 5. D escripción del dominio "C ompetencias del profesorado" con unidades de significado representativas}

\begin{tabular}{|c|c|}
\hline Dominio 2: "Competencias del profesorado" & Unidades de significado representativas \\
\hline $\begin{array}{l}\text { Opiniones con respecto a : } \\
\text { - } \quad \text { Rol como facilitador del aprendizaje y } \\
\text { del ambiente generado en el aula } \\
\text { - Trabajo colaborativo entre pares } \\
\text { - } \quad \text { Dominio metodológico } \\
\text { - Compromiso docente }\end{array}$ & $\begin{array}{l}\text { "el docente es una persona que facilita las cosas, } \\
\text { entrega valores y conductas, cumple normas y } \\
\text { evalúa en consecuencia", "debe motivar siempre" } \\
\text { "son relaciones humanas que se logra establecer } \\
\text { con ellos y confianza" } \\
\text { "buen momento para compartir experiencias entre } \\
\text { tutores" } \\
\text { "las críticas se generan tanto de tutores como de } \\
\text { estudiantes porque no han asumido los verdaderos } \\
\text { roles", "el docente tiene que prepararse, saber a lo } \\
\text { que va, que tema va ha tocar, que es lo que se } \\
\text { pretende, cuales son los resultados y saber como } \\
\text { puede guiar al grupo cuando hay alguien que } \\
\text { queda más atrás o que no participa" } \\
\text { "el conocimiento de los pasos que hay que seguir, } \\
\text { absolutamente se tienen que manejar, el tutor debe } \\
\text { sentirse a gusto participando", "entusiasta y com- } \\
\text { prometido" }\end{array}$ \\
\hline
\end{tabular}

\section{Tabla 6. D escripción del dominio "Aprendizaje centrado en el estudiante" con unidades de significado representativas}

\begin{tabular}{|c|c|}
\hline Dominio 3: "Aprendizaje centrado en el estudiante" & Unidades de significado representativas \\
\hline $\begin{array}{l}\text { Opiniones enmarcadas en las características del } \\
\text { aprendizaje: } \\
\text { - Ambiente de aprendizaje favorable } \\
\text { - Integración de conocimientos } \\
\text { - Aprendizaje activo, significativo y colaborativo } \\
\text { - Relaciones interpersonales }\end{array}$ & $\begin{array}{l}\text { "me ha permitido aprender y al mismo tiempo } \\
\text { pasarlo bien" } \\
\text { "buscar información para tener una compren- } \\
\text { sión integrada", "abarca todos los aspectos" } \\
\text { "preguntan muchísimo más porque ellos ya han } \\
\text { estudiado", "los aprendizajes van a ser más } \\
\text { permanentes", "los alumnos aprendan a través } \\
\text { de situaciones problemáticas que lo motivan a } \\
\text { buscar información permitiéndole una compren- } \\
\text { sión global de la situación" } \\
\text { "hay apoyo del grupo en distintas situaciones, hay } \\
\text { colaboración y se hacen amistades que son acoge- } \\
\text { doras en la experiencia", "establecen una relación } \\
\text { duradera en primer año, se ve en cursos superiores" }\end{array}$ \\
\hline
\end{tabular}

sino el de acoger al estudiante con sus problemáticas y situaciones socio-afectivas, contribuyendo al crecimiento personal de los educandos.
Los entrevistados identifican competencias que deben tener los tutores para trabajar con esta metodología, las cuales se vinculan con la concep- 


\section{Tabla 7. D escripción del dominio "Proceso evaluativo" con unidades de significado representativas}

\begin{tabular}{|c|c|}
\hline Dominio 4: "Proceso evaluativo" & Unidades de significado representativas \\
\hline $\begin{array}{l}\text { Expresiones referidas a la va- } \\
\text { loración de la evaluación del } \\
\text { estudiante (autoevaluación, } \\
\text { evaluación al docente y de } \\
\text { pares) y del docente (evalua- } \\
\text { ción compleja, significativa, } \\
\text { rol de facilitador y desarrollo } \\
\text { de la autoevaluación) }\end{array}$ & $\begin{array}{l}\text { "la autoevaluación es sinónimo de autocrítica, reconoce en sí mismo } \\
\text { lo que está haciendo bien para seguir manteniéndolo e identificar } \\
\text { debilidades para mejorarlas", "cuesta al comienzo evaluar su propio } \\
\text { desempeño" } \\
\text { "cuando alguien me critica creo que es parte del éxito, estoy dando } \\
\text { confianza...", "me gusta recibirlas" } \\
\text { "los pares evalúan a los pares", "son muy críticos con sus compañeros" } \\
\text { "me parece que es difícil para los estudiantes, nunca lo han hecho, } \\
\text { consideran que es algo que debe hacer el docente", "al principio me costó } \\
\text { ya que no era una práctica habitual, luego me di cuenta que es importante } \\
\text { recibir la mirada de otros con respecto a lo que uno está entregando" } \\
\text { "es una manera que nos permite crecer a nosotros y a los alumnos" } \\
\text { "debemos responsabilizarlos de opinar, tienen que sintetizar, re- } \\
\text { flexionar y pensar lo que van a decir para formalizarlo" } \\
\text { "crear las condiciones de critica abierta es una de las tareas difíciles" } \\
\text { "no estamos acostumbrados a evaluarnos", "recién empezamos en este } \\
\text { sistema de autoevaluación, esta cultura es complicada para los adultos" }\end{array}$ \\
\hline
\end{tabular}

tualización del ABP y destrezas para el trabajo en grupos pequeños. Con respecto al proceso de cambio, los tutores lo vivencian en diferentes grados de transición ${ }^{12,13}$. En este aspecto, la transformación no se ha desarrollado en forma lineal, sino más bien ha sido compleja, requiriendo el desarraigo de paradigmas, autorreflexión e introspección del quehacer docente. No sólo se ha debido vencer las resistencias individuales, sino que además se ha requerido un nuevo rol en el proceso de gestión. Para los académicos, el sistema evaluativo es complejo y adquiere una connotación diferente. La evaluación de proceso adquiere igual importancia que la de resultados. Para ello deben fortalecerla como un proceso continuo de mejora, desaprender las prácticas evaluativas tradicionales

\section{REFERENCIAS}

1. Tuning Educational Structures in Europe. Informe Final Proyecto Piloto-Fase 1. Universidad de Deusto - Universidad de Groningen. Edición y Cultura. Sócrates; 2003.

2. Altieri E, Hidalgo C, Ibarra P, Moraga A, Soto L. Propuesta Política de Formación General Universi- e incorporar la autoevaluación, la evaluación de pares y la de los estudiantes en su rol como facilitador. En este aspecto, se contribuye al desarrollo de competencias genéricas interpersonales en relación a la crítica y autocrítica.

Finalmente, esta experiencia educativa ha significado transformar la Facultad de Medicina en una comunidad de aprendizaje, tanto académicos como estudiantes aprenden reflexionando a partir de su propia acción. Los docentes han sido, son y serán los protagonistas de las transformaciones, quienes a través de actitud y voluntad se han constituido en los agentes de cambios, generando ideas, realizando innovaciones, creando un clima adecuado, considerando las resistencias y evaluando el proceso.

dad de La Frontera. Dirección Académica de Pregrado, Vicerrectoría Académica, Universidad de La Frontera, 2007; 4-6.

3. Zerchner. En Cáceres, M. La Formación pedagógica de los profesores universitarios. Una propuesta en la profesionalización del docente. Revista Digital Iberoamericana de Educación; 1983. http://www.campusoei.org/revista/deloslectores/475Caceres.pdf (consultado el 20 diciembre de 2007). 
4. Spark Y, Loucks-Horsley. Models of Staff Development. En Houston, W.R. Handobook of Research on Teacher Education. N. York: MacMillan; 1990; 23450.

5. Barrows HS. How to design a problem-based curriculum for the preclinical years. New York, Springer; 1985; 53-79.

6. CLARK E. La búsqueda de un nuevo modelo educacional. Implicancias y nuevas hipótesis acerca del pensamiento y del aprendizaje. Chile: Cuatro Vientos № 2; 1996; 86-106.

7. DueÑas V. El aprendizaje basado en problemas como enfoque pedagógico en la educación en salud. Colomb Med 2001; 32: 189-96.

8. Rodríguez J. Aprendizaje basado en problemas. México: Panamericana; 2003; 27-36.

9. Cabezas, M, Illesca M, Navarro N, Pantoja ma. Capítulo I Aprendizaje Basado en Problemas. En: Cabalín, D. et al, Diseño de Casos Integrado. Oficina de Educación en Ciencias de la Salud, OFECS, Facultad de Medicina, Universidad de La Frontera. Temuco, Chile; 2006; 5-19.

10. Carretero M. Constructivismo y educación. México: Luis Vives; 1997; 24.

11. Delors J. La educación encierra un tesoro. Informe de la UNESCO de la Comisión Internacional sobre la Educación para el Siglo XXI. Barcelona: Grupo Santillana; 1996; 95-108.

12. Fullan M. The meaning of educational change. New York: Teacher College Press; 1991; 5-6.
13. Fullan M. Research into Educational Innovation. En R. Glatter, M. Preedy y C. Rickes (Eds.). Understanding School Management. Open Univ. Pres, Milton Keynes; 1987; 195-211.

14. SCRIVEn M. Selección de Profesorado. En Millman, J. y Darling-Hammond, L. Manual para la evaluación del profesorado. Madrid: La Muralla; 1997; 105-46.

15. Jiménez B. Evaluación de la docencia. En Jiménez, B. et al: Evaluación de programas, centros y profesores. Madrid: Síntesis Educación; 1999; 173-206.

16. TAYLOR SJ, Bogdan R. Introducción a los métodos cualitativos de investigación. Barcelona: Paidós. 1996; 15-27.

17. CARR W. Hacia una ciencia crítica de la educación. Barcelona: Laertes; 1990; 107-23.

18. Rodríquez G, Gil J, García E. Metodología de la investigación cualitativa. Málaga: Aljibe; 1996; 6177.

19. Arnal J, Del Rincón D, Latorre A. Bases metodológicas de la investigación educativa. Barcelona: Hurtado; 1996; 25-48.

20. StaKe R. Investigación con estudio de casos. Madrid: Morata; 1999; 15-8.

21. Glaser B, Strauss A. En McKernan J, Investigación acción y currículum. Madrid: Morata; 2001; 242.

22. Miles Y Huberman. Aspectos básicos sobre el análisis de datos cualitativos. En Rodríguez G., Gil, J., García, Málaga: Aljibe; 1996; 197-215.

23. Guba, Egon, Lincoln, Yvonna. Naturalist Inquiry: London: Sage; 1885; 37-40. 